\title{
Avaliação e Custos do Subprograma de Controle da Tuberculose no Centro de Saúde Escola de Ribeirão Preto, 1988
}

\author{
Evaluation and Costs of the Tuberculosis Control Program in the \\ University School Health Center of Ribeirão Preto, 1988
}

\author{
Aldaisa C. Forster'; Luciana Halpern' \\ Antonio Ruffino Netto ${ }^{1}$ \& Gabriela B. Tozze ${ }^{1}$
}

FORSTER, A. C.; HALPERN, L.; RUFFINO NETTO, A. \& TOZZE, G. B. Evaluation and Costs
of the Tuberculosis Control Program in the University School Health Center of Ribeirão
Preto, 1988. Cad. Saúde Públ., Rio de Janeiro, 8 (2): 183-189, abr/jun, 1992.

There is a recognized gap between the extensive knowledge on tuberculosis and its epidemiological situation. The ongoing reorganization of Public Health Services in Brazil may increase its eficciency and help in the control of diseases. The present study aims at evaluating the development of the tuberculosis control program and its costs in the area of the University School Health Center, in the city of Ribeirão Preto, during 1988.

Cases of tuberculosis reported in the area of the School Health Center (population around 194,700 inhabitants) were studied according to the costs of laboratory examinations, drigs and salaries.

Deficiencies both in the program implementation and in its results were made evident by the following findings: low coverage (only 61 among the estimated 118 cases); lack of laboratory examinations in $48.3 \%$ of pulmonary cases in adults; recovery and death rates equal to $65.6 \%$ and $13.1 \%$ respectively in all forms of the disease; low cost of laboratory examinations and drugs (about half the expected); costs of US\$276.25 per recovery (60\% above the expected) and US\$ 35.74 per hour of medical work (about 4 times the expected).

Keywords: Tuberculosis; Program Evaluation; Cost Analysis; Outcome and Process Assessment; Primary Health Care

\section{INTRODUÇÃO}

Nas décadas de 50 e 60 , havia grande otimismo com a previsão de que, em futuro próximo, a tuberculose deixaria de ser problema de saúde pública. A tecnologia avançada que essa área acumulou, embora conhecida, todavia não se mostrou suficiente na instrumentalização da política desenvolvida pelos organismos internacionais e nacionais especializados com vistas ao controle e eliminação da doença.

No Brasil, os coeficientes de incidência e mortalidade vêm declinando nos últimos anos.

\footnotetext{
'Departamento de Medicina Social da Faculdade de Medicina de Ribeirão Preto - USP. Av. Bandeirantes, 3900, Ribeirão Preto, SP, 14049-900, Brasil.
}

Em 1986, a taxa de mortalidade por tuberculose foi de 4,33 por 100.000 habitantes e houve 83.731 casos notificados no pais (Ministério da Saúde, 1988).

Nos países subdesenvolvidos, o programa de controle da tuberculose deve integrar-se em um sistema de saúde amplo e descentralizado que atenda às necessidades de saúde da população, evitando-se, assim, a abordagem ineficiente de uma só doença (Pio, 1984). São ainda apontados fatores de administração e organização como importantes para a alteração da situação epidemiológica da tuberculose nesses países (Ruffino Netto, 1990).

Neste momento, a tarefa de administrar os serviços de saúde em processo de integração, impōe a organização de recursos e instrumentos segundo parâmetros reais de regionalização, 
cobertura e referência. Por conseqüência, espera-se um aumento da eficiência do serviço e a diminuição de custos das ações de saúde.

Este estudo pretende conhecer os custos das ações de controle da tuberculose no Centro de Saúde Escola e avaliar o desenvolvimento das atividades programáticas na área de abrangência do mesmo no período de um ano.

\section{MATERIAL E MÉTODOS}

O estudo foi realizado no Centro de Saúde Escola de Ribeirão (CSE) em 1988. Este se localiza no $2^{8}$ subdistrito do município onde vivem cerca de 194.700 pessoas. É composto por três unidades de saúde, prestando assistência ambulatorial de nível primário e de nível secundário, em algumas especialidades.

Foram estudados prontuários médicos de casos de tuberculose notificados na área do CSE, sendo coletadas as seguintes informaçōes: do paciente ( $\mathrm{n}^{2}$ de registro, idade e sexo); do seguimento (data do início, $\mathrm{n}^{2}$ de consultas, tempo de tratamento e condições de saída, forma da doença, exames realizados e medicamentos receitados).

Os custos médios de tratamento e de cura foram calculados considerando-se os salários dos profissionais no programa, os preços dos medicamentos nas faturas da Secretaria de Estado da Saúde, e os preços dos exames laboratoriais constantes das listagens do Ministério da Previdência e Assistência Social em 1988.

\section{RESULTADOS E DISCUSSÃO}

A partir do risco de infeç̧ão tuberculosa na região sudeste, seria estimado o encontro de 118 casos na área de abrangência do CSE. Contudo, foram notificados e confirmados apenas 61 casos (52,0\% dos casos esperados), sendo um dos fatores explicativos a possível ineficácia da busca ativa de casos. Em avaliação das atividades de controle da tuberculose no país, em 1986, constatou-se que $54 \%$ dos casos notificados resultaram de investigação de sintomáticos respiratórios (significando 9,8\% das pessoas examinadas) (Ministério da Saúde, 1988).

$\mathrm{Na}$ Tabela 1 é apresentada a distribuição dos casos de tuberculose em 1988, na área do CSE, segundo a forma e grupo etário. Observa-se que $93,3 \%$ dos casos de tuberculose ocorrem em maiores de 15 anos, dos quais $82,0 \%$ são formas pulmonares, $45,6 \%$, pulmonares sem confirmação baciloscópica e $54,3 \%$, baciliferas; e $18,3 \%$, extrapulmonares. Esta última taxa corresponde à encontrada na regiảo sudeste $(16,0 \%)$ e é maior que as observadas nas demais regiões do país. Isto talvez se deva à acessibilidade a técnicas diagnósticas.

TABELA 1 - Casos de Tuberculose Segundo a Forma e a Faixa Etária, CSE - Ribeirão Preto, 1988

\begin{tabular}{l|cc|ccc}
\hline \hline \multirow{2}{*}{ Faixa } & \multicolumn{5}{|c}{ Formas } \\
\cline { 2 - 6 } Etária & \multicolumn{3}{|c|}{ Pulmonar } & \multicolumn{3}{c}{ Extrapulmonar } \\
\cline { 2 - 6 } & Positivo & Sem Confirmação & Meningite & Outras & Total \\
\hline$<1 \mathrm{a}$ & - & 1 & - & - & 1 \\
$1+5$ & - & 1 & - & - & 1 \\
$5+15$ & - & 1 & - & 1 & 2 \\
$15+20$ & 1 & 1 & - & - & 2 \\
$20+30$ & 7 & 7 & 1 & 1 & 16 \\
$30+40$ & 3 & 6 & - & 1 & 10 \\
$40+50$ & 6 & 5 & - & 3 & 14 \\
$50+60$ & 3 & 5 & - & 3 & 11 \\
$60 \mathrm{e}+$ & 1 & 1 & - & 1 & 3 \\
\hline Total & 21 & $28 *$ & 1 & 10 & 60 \\
\hline \hline
\end{tabular}

* 1 caso com idade desconhecida 
A proporção de casos pulmonares sem confirmação baciloscópica $(45,6 \%)$ entre os adultos é elevada se comparada à esperada, que seria (30\%), segundo Gerhardt (Ruffino Netto, 1990), ou à encontrada no Brasil (1986), que foi de $33 \%$.

Verifica-se certa contradição no programa. Enquanto pacientes de formas extrapulmonares realizaram grande quantidade de exames especializados (graças às instituições de saúde que iniciaram as investigações), de outro lado, pacientes classificados como "pulmonares sem confirmação baciloscópica" deixaram de ser submetidos aos mais elementares exames recomendados pelo programa no país. Isto sugere que alguns dos casos arrolados poderiam não ser de fato tuberculosos.

Observa-se, ainda, que $6,7 \%$ dos casos ocorreram em menores de 15 anos, dos quais $25 \%$ eram formas extrapulmonares (os casos esperados, segundo esquema de Gerhardt, seriam, respectivamente, de $15 \%$ e $25 \%$ ). Ocorreu apenas um caso de meningite tuberculosa em adulto. Supõe-se que este fato seja devido à ampla cobertura da vacinação com BCG em meio à população (da qual $70 \%$ eram menores de um ano em 1988). Estudos anteriores de Ruffino Netto et al., em 1984 e 1985, mostraram coberturas de $83 \%$ e $85 \%$, respectivamente, dos menores de 15 anos na área.
As proporções de cura observadas nos casos pulmonares positivos $(71,4 \%)$ e em todas as formas $(65,6 \%)$ são mais baixas que as referidas em estudo de coorte em 1988, de 80,2\% e $79 \%$, respectivamente (Ministério da Saúde, 1988). Entre os casos pulmonares não confirmados, encontraram-se a menor proporção de curados $(55,2 \%)$ e o maior número de óbitos. No total, a proporção de óbitos $(13,1 \%)$ representa, aproximadamente, quatro vezes àquela da coorte citada $(3,4 \%)$. A este dado alarmante acrescenta-se a alta taxa de abandono observada $(13,1 \%)$ (Tabela 2$)$.

A análise dos casos que evoluíram ao óbito revela que cinco destes não recebiam tratamento específico, sendo apenas notificados por ocasião do atendimento de urgência. Outros três óbitos ocorreram em pacientes portadores de AIDS (dois deles recebiam tratamento; o outro, de 20 anos, sexo feminino, que não recebia tratamento algum, apresentou meningite tuberculosa). $\mathrm{O}$ coeficiente de mortalidade por tuberculose na área do CSE foi de 4,1 (por 100.000 habitantes) (cálculo baseado em notificação), e no Brasil, em 1983, foi de 4,3 (Ministério da Saúde, 1988).

Foram hospitalizados quatro doentes, cuja permanência da internação variou entre dois e seis meses.

TABELA 2 - Casos de Tuberculose Segundo a Forma e a Condição de Saída, CSE - Ribeirão Preto, 1988

\begin{tabular}{|c|c|c|c|c|}
\hline \multirow{3}{*}{ Saída } & \multicolumn{3}{|c|}{ Forma } & \multirow{3}{*}{ Total } \\
\hline & \multicolumn{2}{|c|}{ Pulmonares } & \multirow{2}{*}{ Extrapulmonares } & \\
\hline & Positivos & Sem Confirmação & & \\
\hline Curados & $15(71,4)$ & $16(55,2)$ & $9(81,8)$ & $40++(65,6)$ \\
\hline Abandono & 3 & 4 & 1 & $8 \quad(13,1)$ \\
\hline Transferidos & 1 & 3 & - & $4 \quad(6,6)$ \\
\hline Óbitos & $2+$ & $5+$ & 1 & $8 \quad(13,1)$ \\
\hline Recidiva & - & 1 & - & $1 \quad(1,6)$ \\
\hline Total & 21 & 29 & 11 & 61 \\
\hline
\end{tabular}

+1 óbito devido a AIDS

++2 casos em tratamento prolongado 
Sem dúvida, caberiam aqui as indagações de Penna (1988) sobre as evidências da efetividade epidemiológica dos programas de tuberculose fundamentados em atividades diagnósticas e tratamento de casos.

Os resultados apresentados e discutidos sugerem que a programação não estaria cumprindo a sua meta social, que consistiria em aliviar o sofrimento humano, previnir a invalidez e o óbito por tuberculose (Pio, 1984).

A preocupação é maior ao se considerar que o municipio vem implementando as estratégias da política de saúde (PAIS e rede de serviços de atenção primária) e que estas não resultaram, ao que parece, num melhor efeito da programação.

A análise da operacionalização do programa mostrou 15 pacientes em esquema de quimioprofilaxia, concentração de consultas igual a 6,2 (a norma programática é seis consultas por paciente); a descoberta de um caso pulmonar com baciloscopia negativa entre os comunicantes; 44 comunicantes consultados $(0,72$ comunicante por doente. A estimativa do IBGE para Ribeirão Preto é de 4,6 pessoas por domicllio).
A partir do prontuário médico, foi levantado o total de medicamentos efetivamente prescritos e/ou exames complementares realizados para cada paciente. Conhecendo-se os preços dos medicamentos e/ou dos exames, foi possível calcular o que se denominou de preço efetivamente observado dos medicamentos, exames e tratamentos efetuados.

Por outro lado, a partir das normas do Programa de Controle da Tuberculose, foi possível saber o preço estimado de cada tratamento (medicamentos mais exames), dependendo da forma da doença apresentada pelo paciente.

Assim, para cada paciente, havia sempre dois custos de medicamentos, exames e tratamentos: o valor efetivamente observado e o valor estimado.

A Tabela 3 apresenta os valores de custos observados e estimados para medicamentos, exames e tratamentos (totalizando medicamentos e exames para todos os pacientes).

No que se refere aos medicamentos, os custos totais observados e estimados foram, respectivamente, de US $\$ 2412,78$ e US $\$ 4257,12$, sendo estes sempre mais baixos entre os primeiros.

TABELA 3 - Custos (US\$) dos Medicamentos, Exames Complementares e Tratamentos*

Segundo a Forma Clínica da Doença no Subprograma de Controle da Tuberculose, CSE Ribeirão Preto, 1988.

\begin{tabular}{llrrr}
\hline \hline & & Observados (O) & Esperados (E) & \multicolumn{1}{c}{ (E-O) } \\
\hline \multirow{4}{*}{ Medicamentos } & Pulmonar Bacilifera & 1055,74 & 1520,40 & 464,66 \\
& Pulmonar Sem Confirmação & 944,86 & 1976,52 & 1031,66 \\
& Extrapulmonar & 412,18 & 760,20 & 348,02 \\
\cline { 2 - 5 } Exames & Total & US\$ 2412,78 & US\$ 4257,12 & 1844,34 \\
\cline { 2 - 5 } & Pulmonar Bacilifera & 146,35 & 223,23 & 76,88 \\
& Pulmonar Sem Confirmação & 113,58 & 308,27 & 194,69 \\
& Extrapulmonar & 285,37 & 285,37 & - \\
\cline { 2 - 5 } Tratamentos & Total & US\$ 545,30 & US\$ 816,87 & 271,57 \\
\cline { 2 - 5 } & Pulmonar Bacilífera & 1202,09 & 1743,63 & 541,54 \\
& Pulmonar Sem Confirmação & 1058,44 & 2284,79 & 1226,35 \\
& Extrapulmonar & 697,55 & 1045,57 & 348,02 \\
\cline { 2 - 5 } & Total & US\$ 2958,09 & US\$ 5073,99 & 2115,91 \\
\hline \hline
\end{tabular}

* Medicamentos mais exames complementares

** Excluidos os pacientes transferidos 
Calculando-se as diferenças entre os valores observados e os esperados, nota-se entre os casos pulmonares sem confirmação baciloscópica as maiores diferenças absoluta (US\$ 1031,66 ) e relativa (US\$39,68) por paciente. Uma possível explicação para isso seria que é neste grupo de pacientes que se verificam as maiores taxas de abandono, óbito e falta de tratamento específico.

No tocante aos exames complementares, os custos totais dos exames efetivamente realizados e dos que seriam esperados foram, respectivamente, de US\$ 545,30 e US\$ 816,87 . Verificou-se que a maior diferença entre os custos se deveu, principalmente, à não-realização dos exames preconizados nos casos classificados como "pulmonares sem confirmação". É importante ressaltar que, em um primeiro momento, a análise dos prontuários médicos indicava a realização de exames complementares na maioria dos casos extrapulmonares, optando-se, assim, por considerar os custos esperados como iguais aos observados.
Os custos totais dos tratamento observados e esperados foram, respectivamente, de USS 2958,08 e US\$ 5073,99, mostrando menos investimento no tratamento do paciente que o esperado.

Ainda foram considerados como relativos ao programa (nos custos totais do subprograma) os recursos humanos - as horas dispensadas pelos profissionais ao atendimento, notificação e visitação domiciliária - as taxas de administração e de manutenção, além dos custos com medicamentos e exames complementares (Tabela 4).

Os custos totais observados e esperados do programa (US\$ $11.049,93$ e US\$ $10.479,98$ ) e de cada tratamento (US\$ 181,13 e US\$171,80) são aproximadamente iguais, e a correção se dá, no interior dos cálculos, pela diminuição de 77\% do tempo e do custo do trabalho médico necessários para assistir a 61 pacientes.

Os custos com os recursos humanos representam $64,7 \%$ do que se empregou no programa e deveriam representar $42,5 \%$ do total esperado.

TABELA 4 - Custos (US\$) Totais do Subprograma de Controle de Tuberculose no CSE - Ribeirão Preto, 1988

\begin{tabular}{|c|c|c|c|c|c|c|}
\hline \multirow{4}{*}{ Recursos } & \multicolumn{6}{|c|}{ Atividades Programáticas } \\
\hline & \multicolumn{3}{|c|}{ Observados } & \multicolumn{3}{|c|}{ Esperados } \\
\hline & \multirow{2}{*}{ № Horas } & \multicolumn{2}{|c|}{ Custos (US\$) } & \multirow{2}{*}{$N^{2}$ Horas } & \multicolumn{2}{|c|}{ Custos (US\$) } \\
\hline & & & $\%$ & & & $\mathscr{H}$ \\
\hline Médicos & 460 & 3824,54 & 34,6 & 107 & 889,43 & 8,5 \\
\hline Enfermeiros & 48 & 275,10 & 2,5 & 48 & 275,10 & 2,6 \\
\hline Auxiliares & 604 & 2987,07 & 27,0 & 665 & 3288,74 & 31,4 \\
\hline Exames & - & 545,30 & 4,9 & - & 816,87 & 7,8 \\
\hline Medicamentos & - & 2412,78 & 21,8 & - & 4257,12 & 40,6 \\
\hline Direção e Administração" & - & 502,24 & 4,5 & - & 476,36 & 4,5 \\
\hline Limpeza e Manutenção" & - & 502,24 & 4,5 & - & 476,36 & 4,5 \\
\hline Total & & 11049,93 & 100,0 & & 10479,98 & 100,0 \\
\hline Custo por tratamento & - & 181,13 & & - & 171,80 & \\
\hline Custo por cura & - & 276,25 & & - & 171,80 & \\
\hline Custo por ativ. realizada & 501 & 22,06 & & 774 & 13,54 & \\
\hline Custo por Hora-Médico & 460 & 8,31 & & 107 & 8,31 & \\
\hline
\end{tabular}

* Estimativa $5 \%$ do total 
Nas duas situações, prevalece o investimento em pessoal qualificado, uma vez que o Centro de Saúde Escola é sede do Distrito de Saúde, uma área correspondente a $30 \%$ do município, que conta com um hospital regional (Hospital das Clínicas da Faculdade de Medicina de Ribeirão Preto - Universidade de São Paulo), encarregado das atividades de vigilância epidemiológica.

É interessante observar que os custos estimados dos medicamentos representam $40 \%$ dos investimentos no programa e, portanto, um limitante para o seu desenvolvimento nos países subdesenvolvidos (Pio, 1984).

As diferenças encontradas entre os custos observados e os estimados são, respectivamente, por cura: US\$ 276,25 e US\$ 171,80 ; por atividade realizada (consulta a doentes, comunicantes, quimioprofilaxia e visita domiciliária): US\$ 22,06 e US\$13,54, e podem ser explicadas por um trabalho pouco ativo do CSE na operacionalização do programa.

\section{CONCLUSÕES}

As taxas de cura e de óbitos do total dos casos $(65,6 \%$ e $13,1 \%$, respectivamente) mostraram a ineficácia e a baixa efetividade do programa na população,bem como a indefinição da política local de saúde, que não descentralizou efetivamente as açōes programáticas no município, particularmente as de busca ativa de doentes nas unidades básicas de saúde. Evidentemente, tais achados têm servido de base para discussão junto com instâncias superiores que dirigem as políticas de saúde. Um centro de saúde escola não poderia se omitir em termos de levantar e discutir tais fatos.

Os baixos custos observados com relação a exames diagnósticos e medicamentos (cerca de $50 \%$ dos custos, segundo as normas), contrariamente ao habitual, significaram ineficiência na execução das ações de controle da tuberculose.

Os custos por cura, iguais a US\$ 276,25 (60\% a mais do que o esperado) e por hora-médico efetivamente trabalhada, de US\$ 35,74 (aproximadamente quatro vezes o esperado US\$ 8,31), são alguns dos exemplos do que ocorre na saúde: ao lado de baixos salários dos profissionais, encontram-se atendimentos de alto custo. Estas situaçōes demandam uma política de recursos humanos voltada para a valorização/responsabilidade na qualidade da atenção à saúde.

\section{AGRADECIMENTOS}

Os autores agradecem ao Prof. Dr. Juan S. Yazlle Rocha e José Carlos de Medeiros Pereira pelas observaçōes e sugestōes; à enfermeira Angela Serafim, à Sra. Vera Grine e ao Dr. Artur Watanabe pelo trabalho crítico e empenho em melhorar a execução do programa. Ao Professor Assistente Afonsó Dinis Costa Passos pela versão $\mathrm{em}$ inglês do resumo.

\section{RESUMO}

FORSTER, A. C.; HALPERN, L.; RUFFINO NETTO, A. \& TOZZE, G. B. Avaliação e Custos do Subprograma de Controle da Tuberculose no Centro de Saúde Escola de Ribeirão Preto, 1988. Cad. Saúde Públ., Rio de Janeiro, 8 (2): 183-189, abr/jun, 1992. É sabido o descompasso existente entre o largo conhecimento obtido dos estudos da tuberculose e a sua situação epidemiológica. No Brasil, a reorganização dos serviços públicos de saúde pode aumentar a eficiência das açōes de saúde e o controle das doenças. Assim, pretende-se neste estudo: avaliar o desenvolvimento das atividades programáticas e conhecer os custos destas no controle da tuberculose na área do Centro de Saúde Escola, Ribeirão Preto, no periodo de 1988. Foram estudados casos notificados de tuberculose na área do Centro de Saúde

Escola (população de 194.700 habitantes) e os custos médios de tratamento e de cura segundo os salários dos profissionais do programa, os preços dos medicamentos e dos exames laboratoriais preconizados.

A avaliação evidenciou deficiente operacionalização do programa: baixa cobertura (61 casos/118 estimados); dentre os adultos, dos casos pulmonares sem confirmação baciloscópica, $48,3 \%$ não 
realizaram exames; em todas as formas, as proporções de cura e de óbitos foram, respectivamente, de $65,6 \%$ e $13,1 \%$. Baixos custos observados dos exames diagnósticos e dos medicamentos (cerca de 50\% dos custos, segundo as normas) e custos por cura iguais a US\$ 276,25 (cerca de 60\% maior do esperado) e por hora-médico iguais a US\$ 35,74 (aproximadamente quatro vezes o esperado) mostraram a ineficiência na execução e nos resultados do programa.

Palavras-Chave: Tuberculose; Avaliação de Programa; Análise de Custo; Avaliação de Processo e Resultado; Cuidados Primários

\section{REFERÊNCIAS BIBLIOGRÁFICAS}

MINISTÉRIO DA SAÚDE (DNPS \& CNCT), 1988. Avaliação das Atividades de Controle da Tuberculose realizada no Brasil em 1986. Boletim da Campanha Nacional contra a Tuberculose, 2: 43-56.

PENNA, M. L. F., 1988. Tuberculose: assistência ou controle? Boletim da Campanha Nacional contra a Tuberculose, 2: 5-14.

PIO, A., 1984. El futuro de la lucha antituberculosa - problemas y perspectivas. Boletin de la Oficina Sanitaria Panamericana., 96: 101-118.

RUFFINO NETTO, A., 1990. Tuberculose e a discriminação histórica na saúde. Programa do I Congresso Brasileiro de Epidemiologia, p. 20, Campinas.

RUFFINO NETTO, A.; SANCHES, O.; CARNEO, A. S.; NASCIMENTO, C. S. G.; OLIVEIRA, C. E.; SILVA, E. \& CHIRELLI, M. Q., 1985. Cobertura vacinal em menores de 15 anos residentes em área assistida pelo Centro de Saúde Escola de Ribeirão Preto - SP. In: Anais do Congresso Regional Latino-americano da Associação Internacional de Epidemiologia, p. 29, Ribeirăo Preto. 